\title{
High accuracy simulations of Kerr tails: coordinate dependence and higher multipoles
}

\author{
Manuel Tiglio, ${ }^{1,2}$ Lawrence E. Kidder, ${ }^{3}$ and Saul A. Teukolsky ${ }^{3}$ \\ ${ }^{1}$ Department of Physics and Astronomy, and Center for Computation and Technology, \\ Louisiana State University, Baton Rouge, LA 70803-4001, USA \\ ${ }^{2}$ Department of Physics, and Center for Scientific Computation and Mathematical Modeling, \\ University of Maryland, College Park, MD 20742, USA \\ ${ }^{3}$ Center for Radiophysics and Space Research, Cornell University, Ithaca, NY 14853, USA.
}

\begin{abstract}
We investigate the late time behavior of a scalar field on a fixed Kerr background using a $2+1$ dimensional pseudospectral evolution code. We compare evolutions of pure axisymmetric multipoles in both Kerr-Schild and Boyer-Lindquist coordinates. We find that the late-time power-law decay rate depends upon the slicing of the background, confirming previous theoretical predictions for those decay rates. The accuracy of the numerical evolutions is sufficient to decide unambiguously between competing claims in the literature.
\end{abstract}

PACS numbers: 04.25.D-,04.30.Nk,04.70.Bw,02.70.Hm

\section{INTRODUCTION}

Following the original work of Price [1], the late time behavior of fields propagating on a Schwarzschild black hole has been studied for decades. By now there is a rather complete theoretical picture of it, with compelling supporting numerical evidence. Since in the Schwarzschild case the background geometry is spherically symmetric, perturbations can be expanded in spherical harmonics. Solutions with different multipole numbers $(\ell, m)$ evolve independently. For initial data that is not time symmetric, each of these perturbations decays at late times as $t^{-(2 \ell+3)}$, regardless of the type of perturbation (scalar, gravitational, electromagnetic, etc).

In contrast, our understanding of the late-time decay on a Kerr background is still incomplete. In fact, it has been the subject of controversy over the past decade.

Since in the spinning case the background is not spherically symmetric, additional multipoles are generated during the evolution of an initially pure multipole. The first analytical calculations for the decay rate of these dynamically generated multipoles were done by Barack and Ori [2] and Hod [3]. According to their calculations, the interactions between different multipoles and the black hole angular momentum change the decay rate of each of those multipoles compared to the Schwarzschild case. Furthermore, those calculations also predict that in the Kerr case the late time decay rate is not universal, in the sense that it depends on the type of perturbations.

For example, Hod [3] predicted that a scalar perturbation with an initial pure multipole structure with indices $(\ell, m)$ on a Kerr background in Boyer-Lindquist coordinates would be dominated at late times by the following multipole component and decay:

$$
\begin{aligned}
& \Psi \propto Y^{(\ell, m)} t^{-(2 \ell+3)} \quad \text { if } \ell=m \text { or } \ell=m+1 \\
& \Psi \propto Y^{(\ell=m, m)} t^{-(\ell+m+1)} \quad \text { if } \ell-m \geq 2 \text { (even) } \\
& \Psi \propto Y^{(\ell=m+1, m)} t^{-(\ell+m+2)} \quad \text { if } \ell-m \geq 2 \text { (odd) }
\end{aligned}
$$

In contrast, Burko and Khanna [4] argued in favor of a "simple picture", in which at late times the decay would also be dominated by the lowest multipole that can be generated by mode mixing during the evolution, but that such a mode decays in the same way that it would on a Schwarzschild background. In other words, the authors claimed that the details of the multipole interactions do not affect the late time decay rate. Therefore, according to this simple picture the late time decay for a massless scalar field in a spinning Kerr background should simply be $t^{-\left(2 \ell_{\min }+3\right)}$, where $\ell_{\min }$ is the lowest multipole that can be generated during the evolution. For example, in the axisymmetric case an initial perturbation that is symmetric about the equator would produce even $\ell$ modes by mixing, with $\ell_{\min }=0$. An antisymmetric initial perturbation would have $\ell_{\min }=1$.

The simplest case in which these predictions differ is the axisymmetric solution of the wave equation with $\ell=4$ initial data. The lowest multipole that can be generated during evolution is the monopole term. The simple picture argument predicts for it a decay as in Schwarzschild, $t^{-3}$, while Hod's calculations predict instead a decay of $t^{-5}$. Early numerical studies of the wave equation on a Kerr background in Boyer-Lindquist coordinates by Krivan [5] found an approximate decay of $t^{-5.5}$, which was interpreted by some as an approximate verification of Hod's prediction. Others suggested that Krivan's results were plagued by numerical problems and that Krivan's time decay of $t^{-5.5}$ should not be interpreted as supporting Hod's prediction, but as a numerical artifact, a transient, or both [4]. Krivan in fact, had reported problems with the angular differentiation in the code used in [5]. In particular the decay rate found in [5] does not appear to get closer to $t^{-5}$ with increasing angular resolution but to $t^{-5.5}$, leaving room for questioning the interpretation of the result as a confirmation of Hod's prediction.

In order to settle this issue, Burko and Khanna [4] performed simulations of the wave equation on a Kerr background using "ingoing Kerr" coordinates and obtained a late time decay very close to $t^{-3}$, concluding that Krivan's results were indeed misleading because of numerical artifacts. 
It turns out, however, that the simulations of Burko and Khanna and Krivan do not correspond to the same physical scenario. In the spinning case, the characterization of the initial data in terms of a spherical harmonic decomposition is not unique, since there is no preferred sphere with respect to which such a decomposition should be done. In particular, a pure $\ell=4$ initial perturbation in ingoing Kerr coordinates does not correspond to pure $\ell=4$ initial data in Boyer-Lindquist coordinates, and vice-versa. Not only are the spatial coordinates different, but also the slicing of spacetime. In fact, in a more recent analytic calculation, Poisson [6] explicitly showed that for perturbations of flat spacetime (where, in particular, the contribution due to the spin is treated only to leading order) the decay does depend on the choice of coordinates. Furthermore, Poisson was able to obtain both Hod's and the simple prediction within this linearized scenario, depending on which coordinates are used.

In Ref. 7] Scheel et al. presented high accuracy evolutions of the wave equation on a Kerr background using pure multipole initial data in Kerr-Schild coordinates up to $\ell=4$. Their numerical results confirmed the predictions of the simple picture with high accuracy; in particular, they were consistent with those of Burko and Khanna.

More recently, Gleiser, Price and Pullin [8] have presented numerical results for linear scalar perturbations of a Schwarzschild black hole where the effect of the spin is treated perturbatively. This is done through a hierarchy of one-dimensional evolution equations, which take into account increasingly higher order corrections due to the spin, in what would correspond to Boyer-Lindquist coordinates. Their results show that if one starts with a pure $\ell=4$ mode in those coordinates and solves the corresponding hierarchy of equations to study the late time behavior of the monopole term (which is the one that dominates at late times) at the leading order in the angular momentum, it decays as in Hod's prediction (i.e., as $t^{-5}$ ), not as in the simple picture prediction. The advantage of this approach is that the resulting equations can be solved by a simple numerical code, since they are one-dimensional in space. The disadvantage is that the treatment is perturbative in the black hole angular momentum, leaving open the question of whether the full non-linear dependence on the angular momentum would change the results.

The goal of this paper is to study the late time behavior of fields in a Kerr spacetime by numerically evolving a scalar field in axisymmetry, including the full dependence of the equations on the spin, and unambiguously determine what the decay is, and whether it depends on the coordinates used. For that purpose we numerically evolve a scalar field on a Kerr background, using both Kerr-Schild and Boyer-Lindquist coordinates. Our simulations are of enough accuracy so as to rule out any possibility of numerical artifacts. We discuss the case that has been under dispute, which is the simplest one in which Hod's and the simple prediction disagree, namely pure $\ell=4$ initial data. We also go beyond it and analyze the $\ell=5,6,7,8$ initial data cases.

Our results for the Kerr-Schild case extend those of Scheel et al. and Burko and Khanna to higher accuracy and higher multipole initial data. In particular, we confirm that the decay in those coordinates is governed by the predictions of the "simple picture". For BoyerLindquist coordinates our results extend those of Krivan and of Gleiser, Pullin and Price, again to higher accuracy and higher multipole initial data. Consistent with the conclusions of those references, we unambiguously find that the decay rate in these coordinates is the one predicted by Hod.

The organization of this paper is as follows. In Sec. II we describe the method we use to numerically evolve the scalar field. In Sec. III we present our results for pure $\ell$ initial data for both Kerr-Schild and Boyer-Lindquist coordinates. Finally, we present our conclusions in Sec. IV.

\section{THE NUMERICAL METHOD}

We evolve axisymmetric solutions of the wave equation $\nabla^{a} \nabla_{a} \Psi=0$ on a fixed Kerr black hole background in both Kerr-Schild and Boyer-Lindquist coordinates. In both cases we cast and numerically solve the equations as a first order in space and time system. For example, in the Boyer-Lindquist case we write the equations as

$$
\begin{aligned}
\dot{\Psi}_{t}= & \frac{\left(r^{2}+a^{2}\right)^{2}}{\Sigma^{2}} \partial_{r_{*}} \Psi_{r_{*}}+\frac{2 r \Delta}{\Sigma^{2}} \Psi_{r_{*}}-\frac{2 y \Delta}{\Sigma^{2}} \Psi_{y} \\
& +\frac{\Delta\left(1-y^{2}\right)}{\Sigma^{2}} \partial_{y} \Psi_{y}, \\
\dot{\Psi}_{r_{*}}= & \partial_{r_{*}} \Psi_{t}, \\
\dot{\Psi}_{y}= & \partial_{y} \Psi_{t}, \\
\dot{\Psi}= & \Psi_{t},
\end{aligned}
$$

where $M, a$ are the mass and spin of the black hole, respectively,

$$
\begin{aligned}
\Delta & =r^{2}-2 M r+a^{2} \\
\Sigma & =\left[\left(r^{2}+a^{2}\right)^{2}-a^{2} \Delta\left(1-y^{2}\right)\right]^{1 / 2} \\
y & =-\cos \theta
\end{aligned}
$$

and where we have introduced the Kerr-tortoise coordinate $r_{*}$, defined by

$$
\frac{d r_{*}}{d r}=\frac{r^{2}+a^{2}}{\Delta}
$$

Here $\Psi_{r_{*}}, \Psi_{t}$, and $\Psi_{y}$ are auxiliary variables introduced to cast the system in first order form; at the continuum they satisfy $\Psi_{r_{*}}=\partial_{r_{*}} \Psi, \Psi_{t}=\partial_{t} \Psi$, and $\Psi_{y}=\partial_{y} \Psi$.

We solve the resulting equations using a special purpose two-dimensional code written for this project. A requirement for the numerical solution is that it be demonstrably accurate enough that there is no doubt about the 
results. Since the solution is smooth, a spectral method should be optimal in terms of efficiency for high accuracy. Accordingly, we use a pseudo-spectral collocation (PSC) method in space and the method of lines to evolve in time. A by-product of using a spectral method is that we avoid all difficulties in handling the polar singularities in spherical coordinates.

In the simulations shown below the domain in the radial direction is partitioned into blocks, each of length $10 M$ (in either Boyer-Lindquist-tortoise or Kerr-Schild coordinates). On each block the dependence of the solution in the angular and radial directions is expanded in spherical harmonic and Chebyshev polynomials, respectively, using Gauss-Lobatto collocation points. The solution is advanced in time at each of these points using a fourth-order Runge-Kutta method. Information at the interfaces of the different blocks is communicated through characteristic variables using a penalty method, as described in [9, 10].

We use initial data of the form

$$
\begin{aligned}
\Psi(t=0) & =0 \\
\Psi_{t}(t=0) & =e^{-\left(r-r_{0}\right)^{2} / \sigma^{2}} Y^{(\ell, 0)},
\end{aligned}
$$

where $r_{0}=20 M$ and $\sigma=4 M$. In these expressions as well as in the results shown in the next section, $r$ and $t$ are the Boyer-Lindquist or Kerr-Schild radial and time coordinates, respectively, depending on the equations being solved.

We set the angular momentum to $a=0.5 M$. In the Kerr-Schild case the black hole singularity is excised by placing a purely outflow inner boundary at $1.8 M$, while in the Boyer-Lindquist case the inner boundary is placed at $r_{*}=-40 M$ (which in Boyer-Lindquist radius corresponds to a distance of $\sim 10^{-8} M$ from the event horizon) and we set the incoming modes to zero. In both cases the outer boundary is placed far away enough so that the results here shown are causally disconnected from the type of boundary conditions there imposed (incoming modes set to zero). In more detail, if we evolve for a total time $T$, we typically place the outer boundary at $r=T / 2+100 M+r_{I B}$, where $r_{I B}$ is the radial location of the inner boundary.

The number of collocation points in the radial and angular directions per domain is denoted by $n_{r}, n_{\ell}$, respectively and, unless otherwise stated, the time step is kept fixed at $\Delta t=0.025 \mathrm{M}$. Obtaining fast convergence as we increase the number of collocation points shows that the time step is small enough that the errors due to the time integration are smaller than those associated with the spatial dimensions.

In order to be able to follow the solution for long enough periods of time, especially for the higher multipole initial data cases (which decay faster) we use quadruple precision.

\section{I=4 Kerr-Schild initial data and evolution}

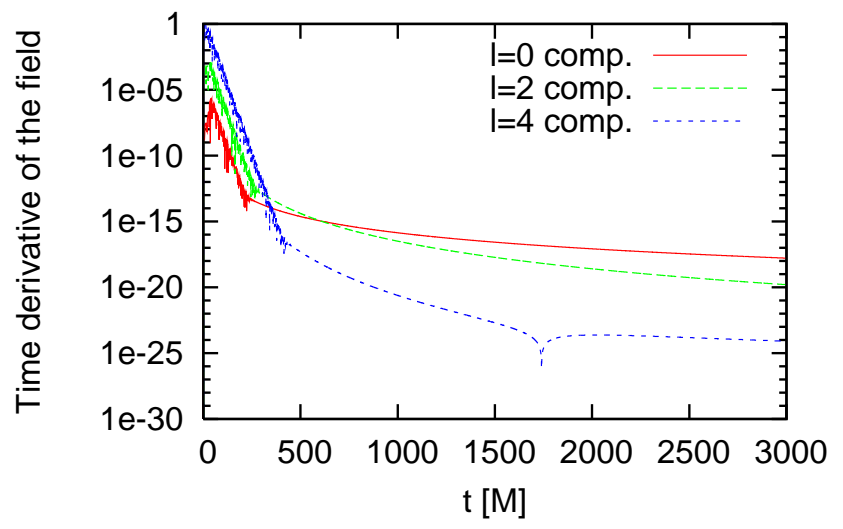

Figure 1: Decay of an initial $\ell=4$ mode in Kerr-Schild coordinates.

\section{RESULTS}

Hod's predictions and the simple one coincide for $\ell=$ $0,1,2,3$ initial data. We have checked that our simulations reproduce the expected decay for each of those values of $\ell$, using both Boyer-Lindquist and Kerr-Schild coordinates. In particular, we have found that $\ell=0$ and $\ell=1$ modes already present in the initial data have a late time decay of $t^{-3}$ and $t^{-5}$, respectively. As we will show below, and as predicted by Hod and Poisson, the decay of these multipoles is different when they are dynamically generated in a Boyer-Lindquist background.

In the following we report our results for higher multipole initial data, for which Hod's prediction and the simple one disagree. We show our results for observers at $r=21.8 M$ in the Kerr-Schild case and $r_{*}=20 M$ in the Boyer-Lindquist one, though similar results hold for other observer locations.

\section{A. The $\ell=4$ case}

Figure 1 shows the decay of the scalar field versus time for a fixed observer radius and $\ell=4$ initial data and evolution in Kerr-Schild coordinates. Shown are the first few $(\ell=0,2,4)$ even multipole components of the solution [the odd components stay at quadruple precision roundoff values $\left(\sim 10^{-35}\right)$ at all times]. Initially only the $\ell=4$ mode is present, but additional modes are generated during evolution; at late times the monopole term dominates.

Figure 2 shows the local power index, defined as $L P I(\ell, t)=-t \Psi_{t}^{(\ell)} / \Psi^{(\ell)}$ (where $\Psi^{(\ell)}$ is the projection of the solution to its $\ell$-multipole component), for the monopole term shown in Fig [1] If the monopole term decays as $\Psi^{(0)} \propto t^{-\mu}$ at late times, then one should obtain that $L P I \rightarrow \mu$. The LPI for the monopole is approaching -3 at late times in our simulations, as predicted by the 


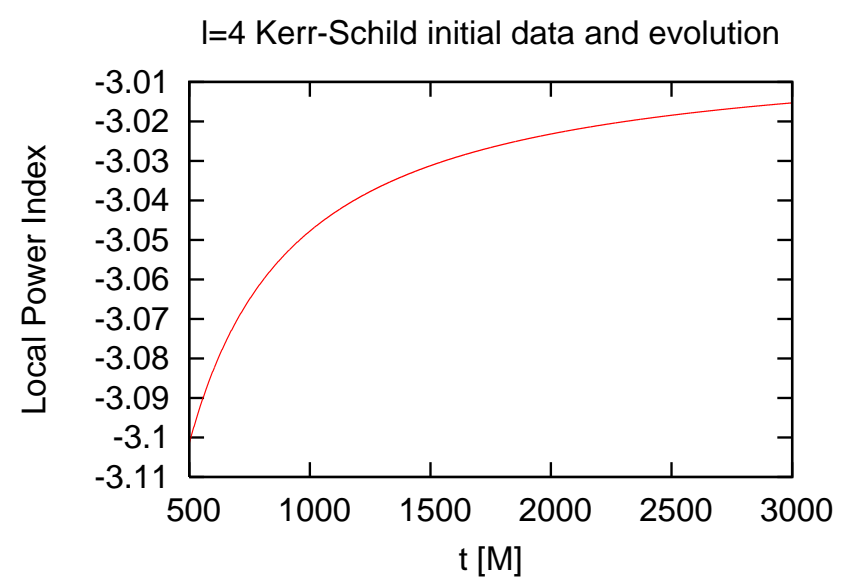

Figure 2: Local power index for the monopole term of the previous figure.

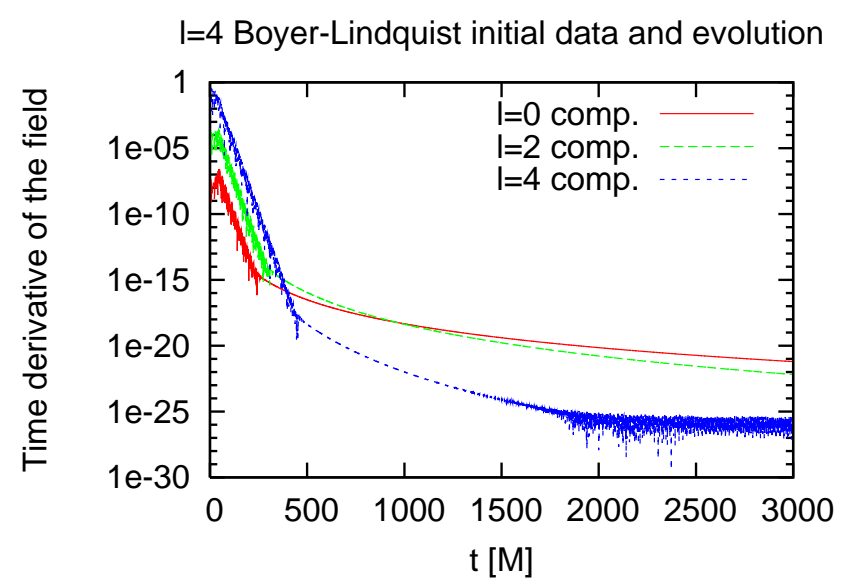

Figure 3: Decay of an initial $\ell=4$ mode in Boyer-Lindquist coordinates.

simple picture.

Figure 3 shows the decay for the evolution of $\ell=4$ initial data in Boyer-Lindquist coordinates. The slowest decaying multipole is again the monopole term, but in this case we find that it decays as $t^{-5}$, as predicted by Hod and shown in Fig. 4 .

The results shown above were obtained with $n_{r}=45$ and $n_{\ell}=4$. To give an idea of the errors in these simulations, Fig. 5 shows the differences in the LPI for the monopole term between different spatial resolutions $\left(n_{r}=30,35,38,40\right)$ and the highest one $\left(n_{r}=45\right)$ as a function of time, keeping $n_{\ell}=4$ fixed. Similarly, the figure also shows the differences in the LPI when keeping $n_{r}=45$ fixed and increasing $n_{\ell}$. At late times the errors introduced by using $n_{\ell}$ Legendre polynomials, where $\ell$ is the multipole index of the initial data (as opposed to using higher order polynomials) is comparable to the errors in the radial direction. Therefore, unless otherwise specified, in the simulations below we use $n_{\ell}$ Legendre polynomials, where the value of $\ell$ is the same as in the

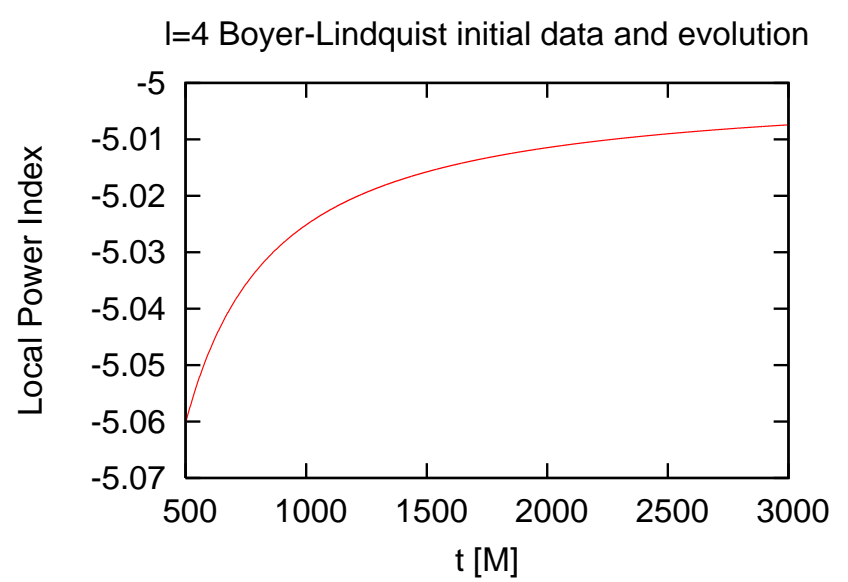

Figure 4: Local power index for the monopole term of the previous figure.

initial data, and $n_{r}=45$.

\section{B. The $\ell=5$ case}

Figure 6] shows our results for the dipole LPI in evolutions of $\ell=5$ initial data in Kerr-Schild and BoyerLindquist coordinates. In both cases the dipole term eventually dominates, with a decay of $t^{-5}$ in the KerrSchild case, as predicted by the "simple picture", and a decay of $t^{-7}$ in Boyer-Lindquist coordinates, as predicted by Hod.

\section{The $\ell=6$ case}

Figure 7 shows our results for the monopole LPI in evolutions of $\ell=6$ initial data in Kerr-Schild and BoyerLindquist coordinates. According to the simple picture interpretation, the monopole term should dominate at late times, with a decay of $t^{-3}$, while Hod's prediction for this case is a decay of $t^{-7}$.

\section{The $\ell=7$ case}

Figure 8 shows our results for the dipole LPI in evolutions of $\ell=7$ initial data in Kerr-Schild and BoyerLindquist coordinates. In the latter case the solution decays much faster and we need to use higher resolution in the radial direction, with an associated smaller timestep for CFL stability. Figure 8 shows our results for both $n_{r}=45, \Delta t=0.025$ and $n_{r}=50, \Delta t=0.0125$. According to the simple picture interpretation, the dipole term should dominate at late times, with a decay of $t^{-5}$, while Hod's prediction for this case is a decay of $t^{-9}$. 

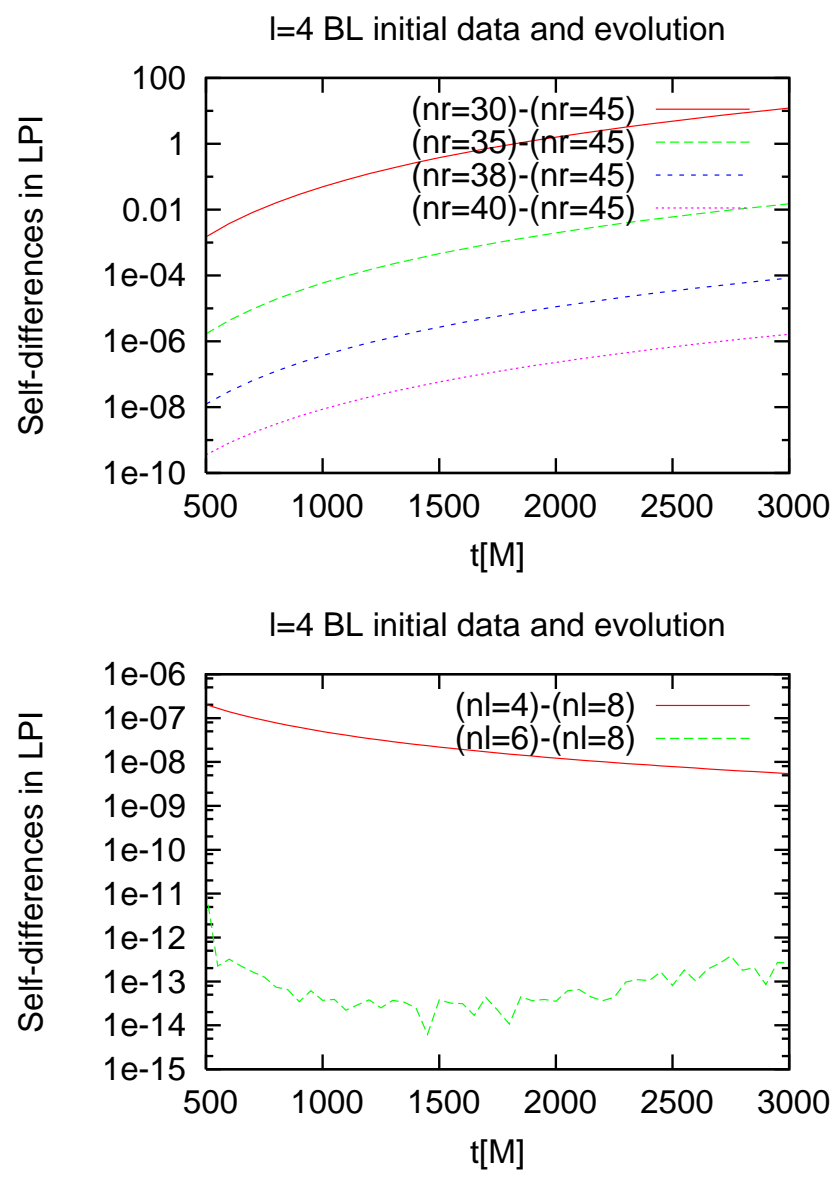

Figure 5: Errors in the LPI for the monopole term of the previous figure.

\section{E. The $\ell=8$ case}

In the Boyer-Lindquist case the monopole term decays even faster for $\ell=8$ initial data and we start reaching quadruple precision roundoff values by the time the tail regime begins, as seen in Figure 9. As a consequence, there is a rather short time interval in which we can measure the local power index, and the latter is not as clean as for the lower multipoles. Figure 10 shows our results for evolutions of $\ell=8$ initial data in both Kerr-Schild and Boyer-Lindquist coordinates. According to the simple picture interpretation, the monopole term should dominate at late times, with a decay of $t^{-3}$, while Hod's prediction for this case is a decay of $t^{-9}$.

\section{DISCUSSION}

In this paper we have evolved an axisymmetric scalar field on a Kerr background using both Boyer-Lindquist and Kerr-Schild coordinates, with pure multipole initial data with indices $\ell=0,1,2 \ldots 6,7,8$, and established the decay rate at late times for each initial data and choice of

\section{I=5 Kerr-Schild initial data and evolution}

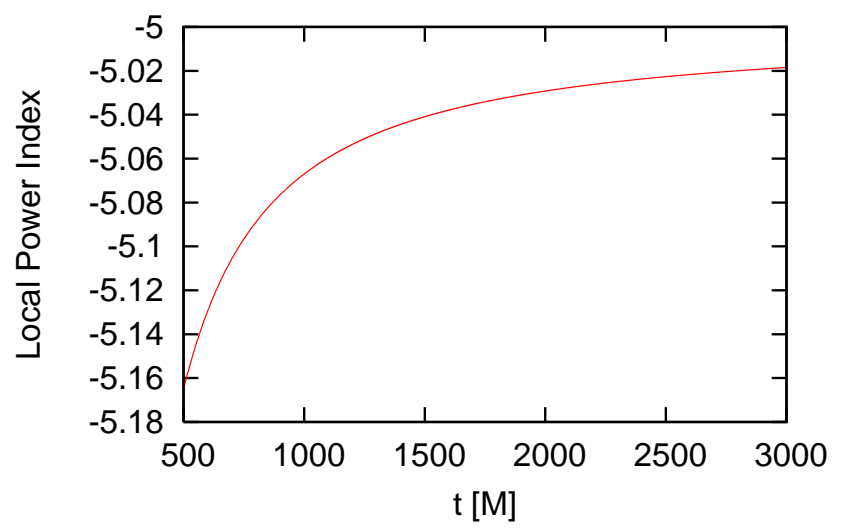

I=5 Boyer-Lindquist initial data and evolution

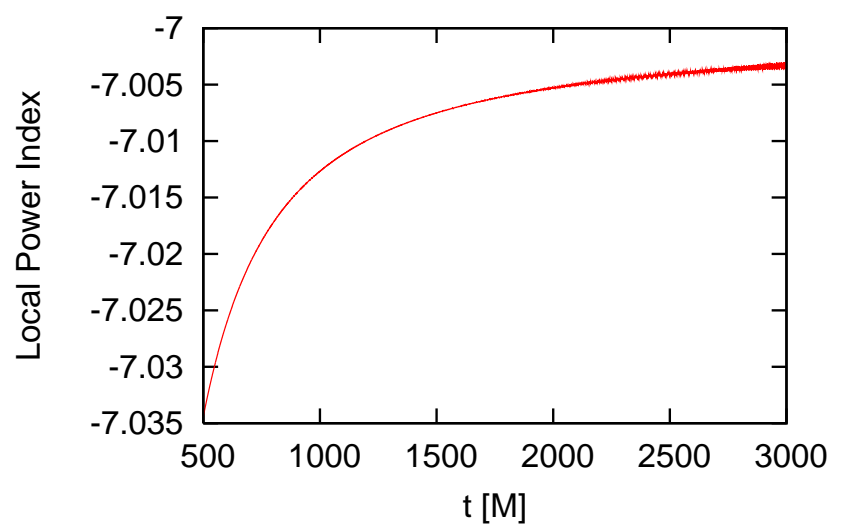

Figure 6: Local power index for the dipole term of $\ell=5$ Kerr-Schild and Boyer-Lindquist initial data and evolution.

background coordinates. The numerical values that we obtained for those rates confirm the "simple picture" prediction [4] in the Kerr-Schild case, but Hod's prediction [3] in the Boyer-Lindquist one. Thus, the most important conclusion of this paper is that the observed latetime decay of the field depends on the time-slicing of the background spacetime.

The differences between the decay rates that we found in our simulations and the above asymptotic $(t \rightarrow \infty)$ predictions are small enough so as to rule out the possibility of numerical artifacts. Those differences are typically less than one percent, except for the $\ell=8$ initial data Boyer-Lindquist case. The dominant factor in these differences in all cases appears to be the fact that we are evolving for a long but finite time; the numerical errors in our simulations are several order of magnitudes smaller. In the particular case of Boyer-Lindquist evolutions of $\ell=8$ initial data, the solution reaches quadruple precision roundoff errors while entering the tail regime. For that reason we cannot determine the decay rate with uncertainties as small as in the $\ell=0 \ldots 7$ cases. The late time decay rate that we find for the $\ell=8$ initial data Boyer-Lindquist case is roughly between -9.2 and 
I=6 Kerr-Schild initial data and evolution

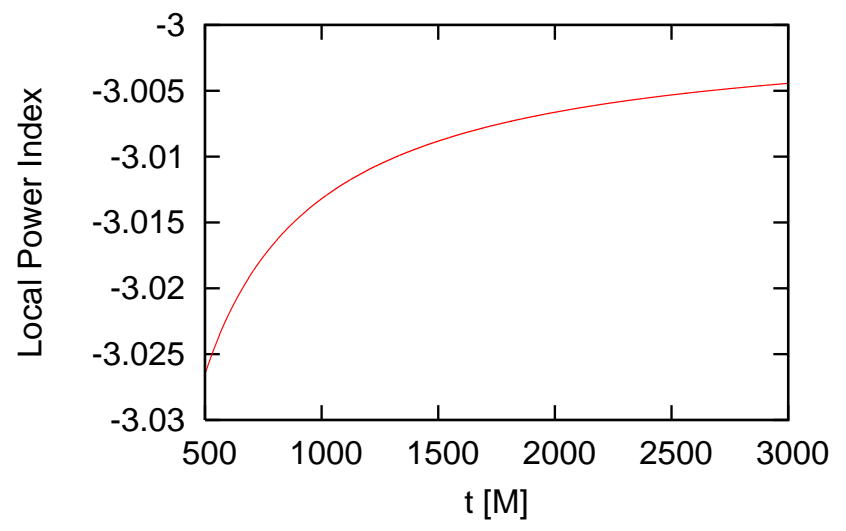

I=6 Boyer-Lindquist initial data and evolution

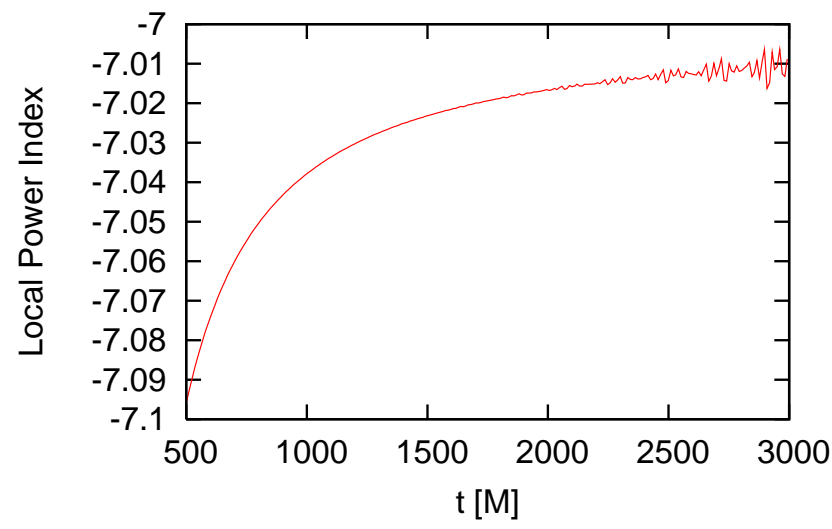

Figure 7: Local power index for the monopole term of $\ell=6$ Kerr-Schild and Boyer-Lindquist initial data and evolution.

-9.0 , to be compared with Hod's prediction $(-9)$ and the simple picture one $(-3)$.

Our results are in apparent, but not real, contradiction with those of Scheel et al. 7] and recent work by Burko and Khanna [11. In order to clarify the source of these apparent contradictions we need to describe parts of those references in some detail.

The 3D code used by Scheel et al. in Ref. [7] only allowed for evolutions on a black hole background where the singularity is excised from the computational domain by placing a purely outflow inner boundary. Since this excludes Boyer-Lindquist coordinates, Kerr-Schild ones $(t, x, y, z)$ were used in Ref. [7]. In this coordinate system, the metric is given by

$$
g_{\mu \nu}=\eta_{\mu \nu}+2 H \ell_{\mu} \ell_{\nu}
$$

where

$$
\begin{aligned}
H & =\frac{M r^{3}}{r^{4}+a^{2} z^{2}}, \\
\ell_{\mu} & =\left\{1, \frac{r x+a y}{r^{2}+a^{2}}, \frac{r y-a x}{r^{2}+a^{2}}, \frac{z}{r}\right\},
\end{aligned}
$$

and the radial coordinate $r$ is defined not as a spherical
I=7 Kerr-Schild initial data and evolution

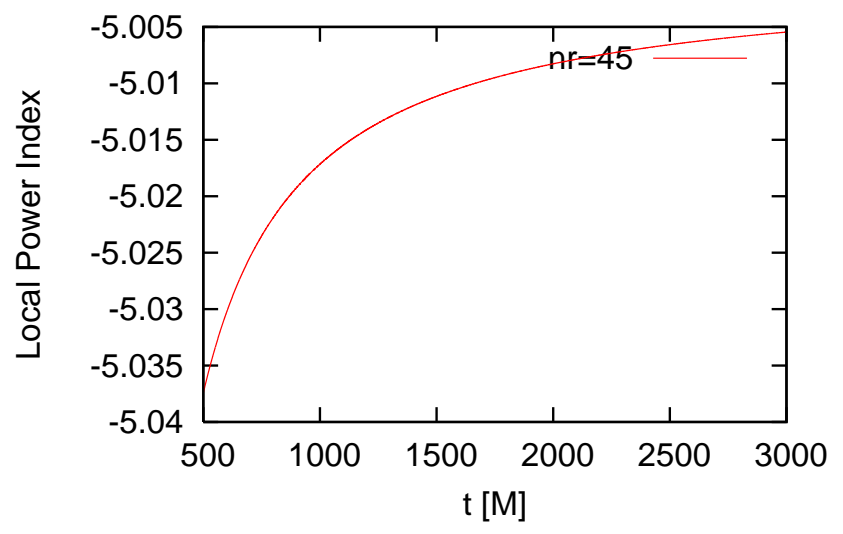

I=7 Boyer-Lindquist initial data and evolution

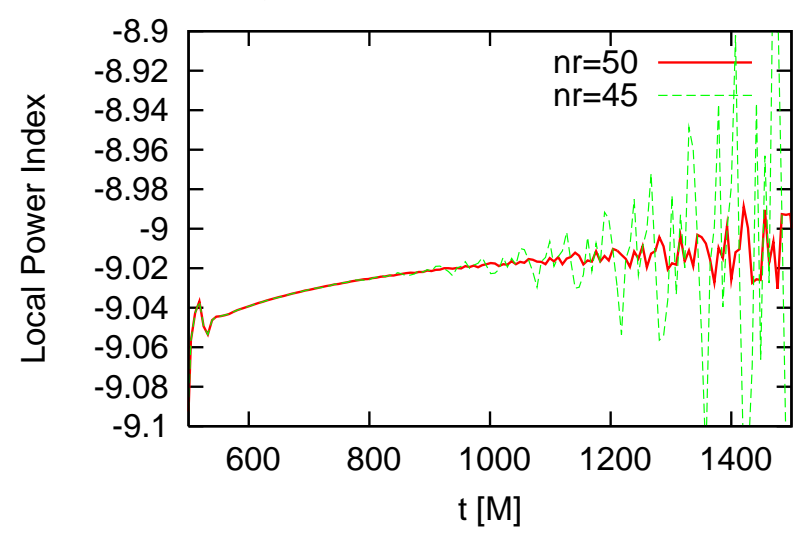

Figure 8: Local power index for the dipole term of $\ell=7$ Kerr-Schild and Boyer-Lindquist initial data and evolution.

coordinate but rather a spheroidal coordinate:

$$
\frac{x^{2}+y^{2}}{r^{2}+a^{2}}+\frac{z^{2}}{r^{2}}=1
$$

Scheel et al. evolved scalar waves on a Kerr-Schild background using two sets of initial data, corresponding to pure multipoles in spherical and spheroidal coordinates on constant-time Kerr-Schild slices. In both cases, they found the tail decayed as in the simple picture. Our Kerr-Schild evolutions confirm this. However, this does not necessarily correspond to the evolution of pure multipole initial data on a constant-time Boyer-Lindquist slice, as seen by a Boyer-Lindquist observer. Hod's prediction is for the late time decay rate seen by an observer with constant Boyer-Lindquist radius as a function of BoyerLindquist time, for an evolution of pure multipole initial data in Boyer-Lindquist coordinates. This is exactly what we have explicitly numerically solved for in this paper.

In more recent work [11], Burko and Khanna evolved pure multipole $\ell=4$ initial data, using Boyer-Lindquist coordinates to define the multipole decomposition as well as to perform the evolution, obtaining a decay of $t^{-3}$, 
I=8 Boyer-Lindquist initial data and evolution

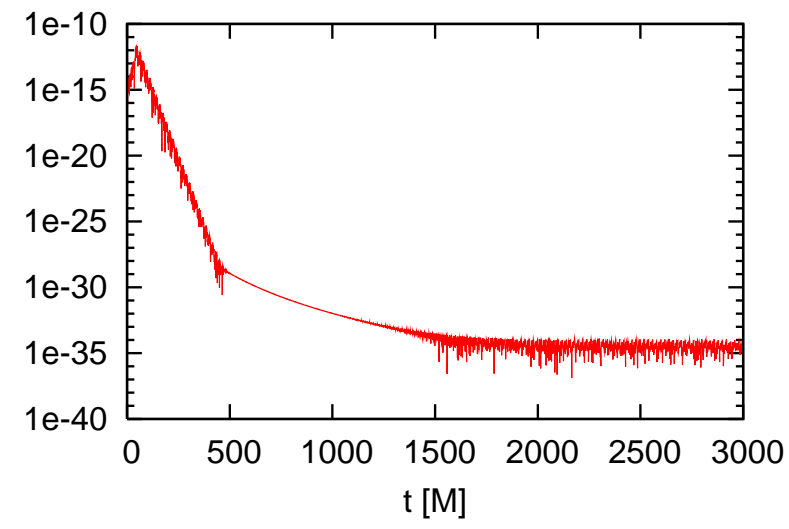

Figure 9: Decay of the monopole term for $\ell=8$ BoyerLindquist initial data and evolution.

which appears to be consistent with the simple picture and in contradiction with our results. The reason for this apparent contradiction seems to be caused by the type of initial data used in Ref. [11]. Rather than giving pure multipole initial data to the scalar field and its time derivative as done in Hod's original work and in this paper, in Ref. [11] pure multipole initial data was given to the scalar field and its "momentum",

$$
\begin{aligned}
\Psi(t=0) & =g(r) Y^{(\ell, m)}, \\
\left(\partial_{t}+b \partial_{r_{*}}\right) \Psi(t=0) & =0
\end{aligned}
$$

where $b=b(r, \theta)$. The angular dependence of $b$ effectively corresponds to adding an $\ell=0$ component to the initial data for $\partial_{t} \Psi$. As explained at the beginning of Sec. III the decay rate of an initial $\ell=0$ mode for the pair $\left(\Psi, \partial_{t} \Psi\right)$ according to both Hod's prediction and the simple picture is $t^{-3}$ (our simulations, as well as previous ones, have in particular confirmed this). What we have found in this paper, though, which is also reported by Gleiser, Price and Pullin [8], is that the decay rate of a dynamically generated monopole in a Boyer-Lindquist coordinates is faster. If as initial data for $\left(\Psi, \partial_{t} \Psi\right)$ one superposes $\ell=4$ and $\ell=0$ modes, as effectively done in Ref. [11], the late time decay rate of each component can be considered independently, since the evolution equations considered are linear. The evolution of the initial $\ell=4$ component will dynamically generate a monopole term that decays as $t^{-5}$, while the evolution of the initial $\ell=0$ component will be dominated by a decay rate of $t^{-3}$. The results of Ref. [11] can be understood by the fact that the latter will dominate over the former at late times.

\section{Acknowledgments}

This research was supported in part by NSF grant PHY 0505761 to Louisiana State University, by a grant

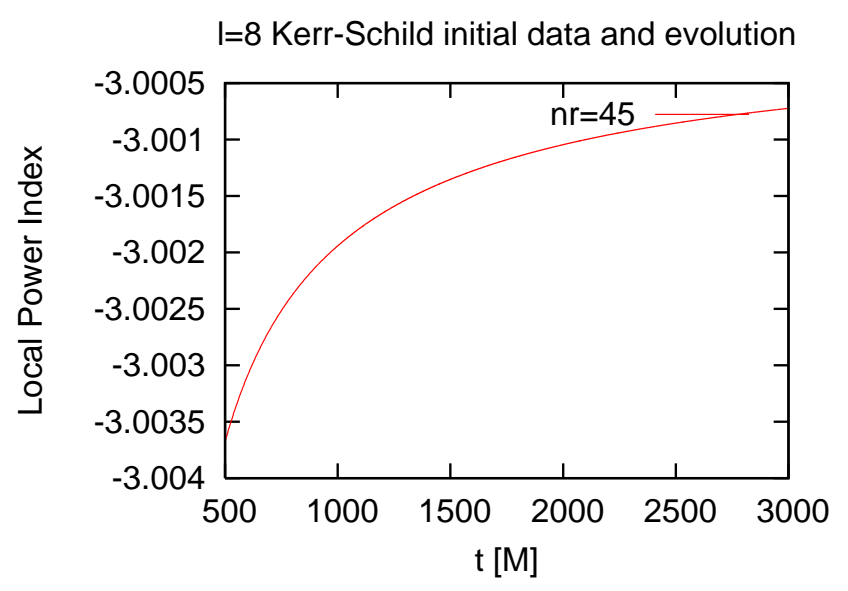

\section{$\mathrm{I}=8$ Boyer-Lindquist initial data and evolution}

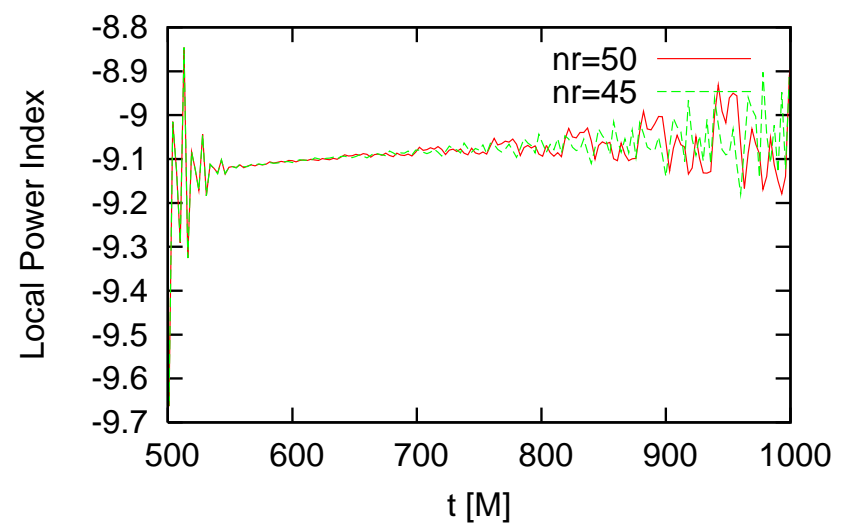

Figure 10: Local power index for the monopole term of $\ell=8$ Kerr-Schild and Boyer-Lindquist initial data and evolution.

from the Sherman Fairchild Foundation to Cornell, and by NSF grants PHY-0652952, DMS-0553677, PHY0652929, and NASA grant NNG05GG51G at Cornell. The research employed the resources of the CCT at LSU, which is supported by funding from the Louisiana legislature's Information Technology Initiative.

MT would like to thank Enrique Pazos and Jorge Pullin for helpful discussions throughout this project.
[1] R. H. Price, Phys. Rev. D 5, 2419 (1972).

[2] L. Barack and A. Ori, Phys. Rev. Lett. 82, 4388 (1999).

[3] S. Hod, Phys. Rev. D61, 024033 (2000).
[4] L. M. Burko and G. Khanna, Phys. Rev. D 67, 081502 (2003).

[5] W. Krivan, Phys. Rev. D 60, 101501 (1999). 
[6] E. Poisson, Phys. Rev. D 66, 044088 (2002).

[7] M. A. Scheel, A. L. Erickcek, L. M. Burko, L. E. Kidder, H. P. Pfeiffer, and S. A. Teukolsky, Phys. Rev. D 69, 104006 (2004), gr-qc/0305027.

[8] R. J. Gleiser, R. P. Price, and J. Pullin (2007), grqc/0710.4183.
[9] J. S. Hesthaven, SIAM J. Sci. Comput. 18, 658 (1997)

[10] J. S. Hesthaven, SIAM J. Sci. Comput. 20, 62 (1999).

[11] L. M. Burko and G. Khanna (2007), arXiv:0711.0960[grqc]. 